\title{
Cluster Based Coordinated Beamforming and Power Allocation for MIMO Heterogeneous Networks
}

\author{
Kianoush Hosseini, Wei Yu, Raviraj S. Adve \\ The Edward S. Rogers Sr. Department of Electrical and Computer Engineering, University of Toronto \\ 10 King's College Road, Toronto, ON M5S 3G4, Canada \\ Email: \{kianoush, weiyu, rsadve\}@comm.utoronto.ca
}

\begin{abstract}
Coordinated intercell interference management is essential in dense heterogeneous networks with limited backhaul capacity. This paper proposes a cluster-based hierarchical cooperative transmission and resource allocation scheme with proportionally fair objective in a cellular network where both the macro base station (BS) and the small cell access-points (SCAs) are equipped with multiple antennas and share the entire available bandwidth. As the first step, SCAs form clusters based on their pairwise distances where each cluster comprised of adjacent SCAs which are potentially strong interferers. Clustering enables intra-cluster coordinated transmission and inter-cluster coordinated resource allocation. Specifically, SCAs within each cluster form a network multiple-input multiple-output (MIMO) system, share the users' data symbols, and cancel intra-cluster interference via zero-forcing spatial multiplexing. Further, a distributed power control scheme is devised for the purpose of mitigating inter-cluster interference without exchanging users' data signals. We show that clustering facilitates intra-cluster coordination by enabling data exchange and channel training with reasonable backhaul communication within each cluster. We also show that the proposed inter-cluster power control scheme can further improve the network-wide utility.
\end{abstract}

\section{INTRODUCTION}

Network densification through employing a large number of distributed small cells (SCs) is a promising approach to satisfy the required data rates of future wireless applications [1]. While the macro base-stations (BSs) serve highly mobile users and extend cell coverage area, SCs can potentially provide local capacity enhancements. However, intercell interference is a dominant limiting factor and can potentially exacerbate the overall network performance in a dense two-tier architecture. Although coordinating all distributed antennas within a cell region via forming a very large virtual antenna array achieves an interference-free operating point, it incurs high data-rate backhaul communications and a prohibitive overhead for channel training. Therefore, devising distributed interference management schemes based on clustering and exploiting local information is desirable.

This paper evaluates the advantages of SC clustering in a downlink of a dense heterogeneous network wherein the macro BS and the SC access points (SCAs) are equipped with multiple antennas. We argue that, given reasonable cluster sizes, each cluster can form a virtual multiple-input multipleoutput (MIMO) network wherein user equipments' (UE) communications are separated via spatial multiplexing using jointly designed downlink precoding vectors. The benefits of this approach is that the intra-cluster interference is completely removed. Further, since only a small fraction of active SCAs form each cooperative cluster, the resulting overhead and complexity is manageable. Moreover, to fully control inter-cluster interference, this paper assumes an architecture in which clusters can coordinate their transmit power via exchanging low-rate messages over the backhaul without sharing the UEs' data symbols.

Coordinated transmission strategies have been proposed for cellular networks without taking clustering into account [2], with fixed clusters [3], [4], and with adaptive clusters [5], [6]. Furthermore, a joint clustering and coordinated transmission strategy is proposed in [7]. However, since the group of serving SCAs for each UE varies with instantaneous channel realizations, the resulting number of required handoffs is prohibitive. Similarity-based clustering schemes have been extensively studied in the context of machine learning, e.g., $k$-means [8], spectral clustering (SP-clusustering) [9], and affinity propagation clustering (AP-clustering) [10], and cooperative game theory [11].

The main contributions of this paper are as follow. Building upon the work [6], we propose two SC clustering schemes based on $k$-means and AP-clustering algorithms in a heterogeneous network. The proposed similarity measure is merely a function of pairwise distances between SCAs. Hence, the cluster formation process is not susceptible to instantaneous channel variations and is stable over long time scales. Due to the small number of SCAs within each cluster, exchanging UEs' data symbols within each group and acquiring channel state information are feasible. Hence, the intra-cluster interference can be completely cancelled by designing downlink zero-forcing beamforming (ZFBF) vectors. Furthermore, using the idea of interference pricing power control [12], we devise a simple inter-cluster coordination scheme which only relies on exchanging simple low-rate messages amongst cluster representatives. We show that SC clustering in heterogeneous networks strikes a balance between the network-wide performance and required backhaul capacity. In particular, clustering renders the coordinated transmission within each cluster viable and the proposed power control scheme provides significant performance gains. Our simulation results show that the proposed approach outperforms conventional percell processing. Moreover, it enables partial interference 


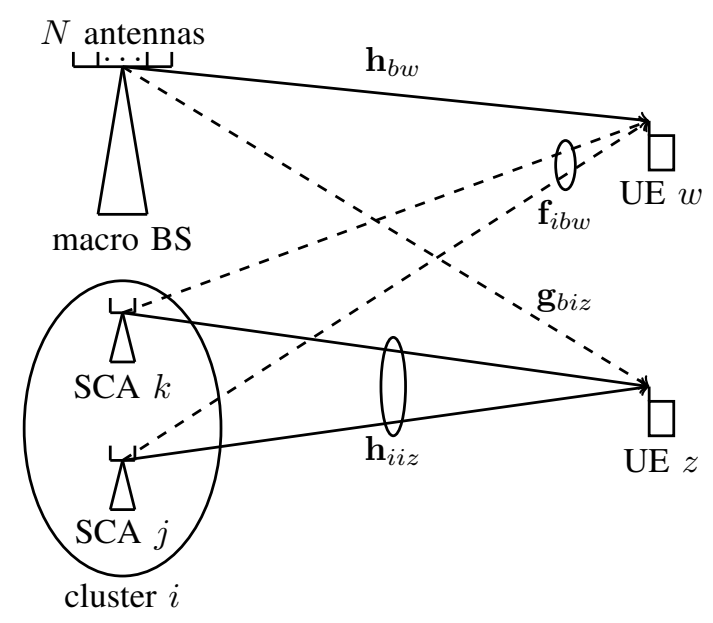

Fig. 1. A cellular network overlaid with two SCAs. Solid arrows denote the direct channels and dashed arrows denote the interfering channels.

cancellation while requiring significantly less backhaul communications as compared to coordinating all active transmitters. It is noteworthy that the key step here is SC clustering that facilitates interference coordination across all transmitters with reasonable complexity and overhead.

\section{Problem Statement}

Consider a two-tier single-cell wireless network where a macro-tier is augmented with $S$ low-power SCAs as schematically shown in Fig. 1. Both tiers transmit concurrently over the shared spectrum of a fixed bandwidth $W$. The macro BS employs $N$ transmit antennas and each SCA $i$ is equipped with $N_{i}$ antennas. We assume that $K$ single-antenna UEs are randomly distributed within the cell region. Furthermore, let $\mathcal{C}=\left\{C_{1}, \ldots, C_{m}\right\}$ be a partition of a SC-tier such that

$$
\begin{gathered}
C_{i} \cap C_{j}=\emptyset \\
\cup C_{i}=S .
\end{gathered}
$$

Each UE measures its received power from all access points and is assigned to the one that provides the highest signalto-interference-and-noise power ratio (SINR). ${ }^{1}$ Let $\mathcal{S}_{i}$ and $\mathcal{U}_{b}$ be the set of UEs associated with SCA $i$ and the macro BS, respectively. Moreover, $\mathcal{U}_{i}^{c}=\cup_{j \in C_{i}} \mathcal{S}_{j}$ represents the set of UEs assigned to SCAs in cluster $i$.

This paper considers an architecture in which the SCAs are partitioned into clusters, and each cluster forms a network MIMO system where the data symbols of its UEs are shared amongst all SCA members. Therefore, each cluster is responsible for performing the following three tasks. First, cluster $i$ employs $M_{i}=\sum_{j \in C_{i}} N_{j}$ transmit antennas and enables $M_{i}$ independent data streams to be communicated to its associated UEs. Therefore, at most $M_{i}$ UEs should be chosen to be served at a given time slot. Second, given the available number of

\footnotetext{
${ }^{1}$ At this step, each access point generates an omnidirectional beam pattern. If no $\mathrm{UE}$ is associated with an access-point, it remains silent.
}

antennas per cluster, downlink beamforming vectors should be designed. Moreover, in order to minimize the intercell interference, appropriate amount of power needs to be allocated to each active beam across the network.

This paper adopts a log-utility objective which maximizes the sum rate while ensuring proportionally fair resource allocation across the UEs. The optimization problem is that of choosing the optimal partition $\mathcal{C}$, scheduling policy, beamforming vectors, and power allocation

$$
\operatorname{maximize} \sum_{i \in \mathcal{C}} \sum_{k \in \mathcal{U}_{i}^{c}} \log _{2}\left(\bar{R}_{i k}^{c}\right)+\sum_{k \in \mathcal{U}_{b}} \log _{2}\left(\bar{R}_{k}^{b}\right)
$$

subject to

$$
\begin{aligned}
& c_{1}: \operatorname{tr}\left[\left(\mathbf{W}_{i}^{c}\right)^{\mathrm{H}} \mathbf{W}_{i}^{c}\right] \leq P_{i}^{c}, \quad\left\{i \mid C_{i} \in \mathcal{C}\right\} \\
& c_{2}: \operatorname{tr}\left[\left(\mathbf{W}_{b}\right)^{\mathrm{H}} \mathbf{W}_{b}\right] \leq P_{b} \\
& c_{3}:(1)-(2) .
\end{aligned}
$$

wherein $\bar{R}_{i, k}^{c}$ and $\bar{R}_{k}^{b}$ are exponentially weighted average rates of UE $k$ associated with cluster $i$ and UE $k$ associated with the macro BS, respectively. Let $K_{i}{ }^{c}$ and $K^{b}$ be the number of scheduled users at cluster $i$ and the macro BS in order. Assuming downlink ZFBF, the precoding matrix of cluster $i$ and the macro BS are given, respectively, as $\mathbf{W}_{i}^{c}=$ $\left[\mathbf{w}_{i 1}^{c}, \ldots, \mathbf{w}_{i K_{i}^{c}}^{c}\right] \in \mathbb{C}^{M_{i} \times K_{i}^{c}}$ and $\mathbf{W}_{b}=\left[\mathbf{w}_{1}^{b}, \ldots, \mathbf{w}_{K_{b}}^{b}\right] \in$ $\mathbb{C}^{N \times K_{b}}$ where $\mathbf{w}_{i k}^{c}$ is the beam vector assigned to UE $k$ by cluster $i$ and $\mathbf{w}_{j}^{b}$ is the beam vector assigned to UE $j$ by the macro $\mathrm{BS}, P_{i}^{c}$ is the maximum available power across the antennas of cluster $i$ and $P_{b}$ denotes the maximum available power at the macro BS. ${ }^{2}$ Moreover, the instantaneous achievable rates of UE $k$ associated with cluster $i$ and the macro BS are as follows

$$
\begin{aligned}
R_{i k}^{c} & =\log _{2}\left(1+\operatorname{SINR}_{i k}^{c}\right) \\
R_{k}^{b} & =\log _{2}\left(1+\operatorname{SINR}_{k}^{b}\right)
\end{aligned}
$$

wherein

$$
\begin{aligned}
\operatorname{SINR}_{i k}^{c} & =\frac{\left|\mathbf{h}_{i i k}^{\mathrm{H}} \mathbf{w}_{i k}^{c}\right|^{2}}{\sum_{\substack{j \neq i \\
m \in \mathcal{U}_{j}^{c}}}\left|\mathbf{h}_{j i k}^{\mathrm{H}} \mathbf{w}_{j m}^{c}\right|^{2}+\sum_{m \in \mathcal{U}_{b}}\left|\mathbf{g}_{b i k}^{\mathrm{H}} \mathbf{w}_{m}^{b}\right|^{2}+N_{o}} \\
\operatorname{SINR}_{k}^{b} & =\frac{\left|\mathbf{h}_{b k}^{\mathrm{H}} \mathbf{w}_{k}^{b}\right|^{2}}{\sum_{j} \sum_{m \in \mathcal{U}_{j}^{c}}\left|\mathbf{f}_{j b k}^{\mathrm{H}} \mathbf{w}_{j m}^{c}\right|^{2}+N_{o}} .
\end{aligned}
$$

$\mathbf{h}_{j i k} \in \mathbb{C}^{M_{j}}$ and $\mathbf{f}_{j b k} \in \mathbb{C}^{M_{j}}$ denote the channel gains between cluster $j$ and UE $k$ associated with cluster $i$ and UE $k$ associated with the macro BS. Furthermore, $\mathbf{g}_{b i k} \in \mathbb{C}^{N}$ and $\mathbf{h}_{b k} \in \mathbb{C}^{N}$ are, respectively, defined as the channel gains between the BS and UE $k$ of cluster $i$ and its own UE $k . N_{o}$ is the noise power at each of the UE antennas.

The optimization problem formulated in (3)-(6) is, in general, non-convex. This paper proposes a hierarchical approach to obtain a local solution in an efficient manner.

\footnotetext{
${ }^{2}$ We impose sum power constraint per cluster.
} 


\section{HiERARCHICAL Algorithm}

This section proposes a hierarchical approach to acquire a practical, albeit local, solution to the optimization problem in (3)-(6). In this regard, we first utilize the $k$-means or the AP-clustering algorithms to find optimized partition of the SCtier based merely on SCA distances. Given fixed clusters, UE scheduling, beamforming, and power spectrum management steps are carried out in order.

\section{A. Clustering}

As mentioned earlier, joint processing within each cluster can be executed in order to cancel intra-cluster interference. Therefore, it is favorable to group those SCAs which potentially impose the strongest interference on UEs of each other, i.e., adjacent SCAs, in order to remove the dominant interfering sources. This section explores two different SC clustering schemes, namely, $k$-means and AP-clustering, and compares their performance and complexity against the previously proposed SP-clustering scheme [9].

1) AP-clustering: Define $s_{i j}=1 / d_{i j}$ to be the similarity measure between SCA $i$ and $j$ where $d_{i j}$ denotes their distance from each other. In order to group the strongest interferers, the highly similar SCAs should share a cluster. Furthermore, any clustering scheme must satisfy the constraints expressed in (1)-(2). Based on the idea of AP-clustering, the clustering optimization problem can be formulated as

$$
\begin{aligned}
& \operatorname{maximize} \sum_{i, j \in \mathcal{S}} s_{i j} c_{i j} \\
& \text { subject to } \\
& \qquad \begin{array}{l}
c_{1}: \sum_{j \in \mathcal{S}} c_{i j}=1, \quad \forall i \\
c_{2}: c_{i j} \in\{0,1\} \\
c_{3}: 0 \leq c_{i j} \leq c_{j j}, \quad \forall i \neq j
\end{array}
\end{aligned}
$$

where $\mathcal{S}$ is the index set of all active SCAs, $c_{i j}$ is an integer membership variable, i.e., $c_{i j}=1$ indicates that SCA $i$ lies in a cluster where SCA $j$ is its clusterhead, constraints $c_{1}$ and $c_{2}$ ensure that the resulting clusters are non-overlapping, and the constraint $c_{3}$ states that SCA $j$ is eligible to be a clusterhead only if it represents itself, i.e., $c_{i j}=0$ if $c_{j j}=0$. The clustering optimization problem is a combinatorial problem. By incorporating the constraints into the objective function, it is possible to modify the problem as an instance of the maxsum problem [13] for which a local maxima can be acquired through exchanging simple messages over the edges of the network factor graph. We refer the readers to [10] and [6] for more details.

2) $k$-means Clustering: $k$-means algorithm is one of the classic clustering methods that partitions the SCAs into $k$ groups in order to minimize the within-cluster sum of distances, i.e.

$$
\mathcal{C}=\arg \min _{C_{1}, \ldots, C_{k}} \sum_{i=1}^{k} \sum_{\left\{j \mid S_{j} \in C_{i}\right\}}\left\|S_{j}-\mathbf{x}_{i}\right\|^{2}
$$

where $\mathbf{x}_{i}$ is the centroid of cluster $i$. The algorithm is initialized by choosing $k$ random centroids. Each SCA measures its distance to all centroid points and joins the closest cluster. Then, the cluster centroids are updated based on the locations of their members. This process iterates until the algorithm converges.

3) SP-clustering: Let $G=(V, E)$ be the similarity graph of the SC-tier, where $V=\left\{v_{1}, \ldots, v_{S}\right\}$ is the set of vertices representing SCAs, and $E=\left\{e_{i j}\right\}_{i, j}$ is the set of edges with a weight representing the similarity $s_{i j}$ between SCA $i$ and $j$. Moreover, let $L=A-D$ be the graph Laplacian matrix of the network where $A \in \mathbb{R}^{S \times S}$ is the similarity matrix and $D=\operatorname{diag}\left\{d_{1}, \ldots, d_{S}\right\}$ where $d_{i}$ is the sum similarity of SCA $i$ to all other SCAs. SP-clustering aims at finding the partition of the SC-tier in order to maximizing the sum of the within-cluster similarities and minimizing the inter-cluster similarities. The work [9] shows that this is equivalent to a trace minimization problem and the solution is characterized by finding $k$ eigenvectors of $L$ associated with the $k$ smallest eigenvalues.

4) Performance v.s. Complexity: The clustering problem is NP-hard and both AP-clustering and $k$-means can only guarantee local optimality. The per iteration complexity of both schemes grows as $\mathcal{O}\left(S^{2}\right)$. Moreover, as it is shown in Section IV, the performance and required backhaul capacity of both schemes are essentially identical. However, there are some fundamental differences between these two schemes. In particular, $k$-means requires the number of clusters to be chosen a priori. However, there is no obvious way to characterize the effect of choosing different values of $k$ on the ultimate performance of the proposed algorithm in advance. Furthermore, $k$-means relies on the geographical locations of the SCAs. Assuming that each SCA has access to GPS information, SCAs' positions need to be exchanged across the network and each SCA joins the cluster to which it has the minimum distance. On the other hand, AP-clustering does not choose the number of clusters a priori. Further, cluster sizes can be controlled by adjusting the self-similarity measures. Moreover, similarity values should be exchanged amongst SCAs in a synchronized fashion.

The "un-normalized" SP-clustering is employed in [5] to group macro BSs in a conventional cellular network. However, as discussed in [9], this type of SP-clustering scheme depends on the number of vertices within each cluster (and not on the actual similarity values). Hence, the acquired clusters may not have comparable sizes, i.e., the un-normalized SP-clustering may form a few number of clusters which contain a large fraction of SCAs, while the remaining SCAs are isolated. Thus, as our simulation results confirm, to enable joint downlink processing within each cluster, this scheme may require high data-rate backhaul links. Moreover, SP-clustering requires a central unit which has access to all pairwise similarity values.

\section{B. Proportionally Fair Scheduling}

Given a fixed user assignment for each cluster, an essential question is that which UEs should be served by the avail- 


$$
\begin{aligned}
t_{i k}^{c} & =\sum_{n \in \mathcal{U}_{b}} \gamma_{n}^{b} \frac{P_{n}^{b}\left|\mathbf{h}_{b n}^{\mathrm{H}} \mathbf{w}_{n}^{b}\right|^{2}\left|\mathbf{f}_{i b n}^{\mathrm{H}} \mathbf{w}_{i k}^{c}\right|^{2}}{\left.\Phi+P_{n}^{b}\left|\mathbf{h}_{b n}^{\mathrm{H}} \mathbf{w}_{n}^{b}\right|^{2}\right)}+\sum_{\substack{q \neq i \\
r \in \mathcal{U}_{q}^{c}}} \gamma_{q r}^{c} \frac{P_{q r}^{c}\left|\mathbf{h}_{q q r}^{\mathrm{H}} \mathbf{w}_{q r}^{c}\right|^{2}\left|\mathbf{h}_{i q r}^{\mathrm{H}} \mathbf{w}_{i k}^{c}\right|^{2}}{\Psi\left(\Psi+P_{q r}^{c}\left|\mathbf{h}_{q q r}^{\mathrm{H}} \mathbf{w}_{q r}^{c}\right|^{2}\right)} \\
t_{k}^{b} & =\sum_{q} \sum_{r \in \mathcal{U}_{q}^{c}} \gamma_{q r}^{c} \frac{P_{q r}^{c}\left|\mathbf{h}_{q q r}^{\mathrm{H}} \mathbf{w}_{q r}^{c}\right|^{2}\left|\mathbf{g}_{b q r}^{\mathrm{H}} \mathbf{w}_{k}^{b}\right|^{2}}{\Psi\left(\Psi+P_{q r}^{c}\left|\mathbf{h}_{q q r}^{\mathrm{H}} \mathbf{w}_{q r}^{c}\right|^{2}\right)} \\
\Phi & =\sum_{j} \sum_{m \in \mathcal{U}_{j}^{c}} P_{j m}^{c}\left|\mathbf{f}_{j b n}^{\mathrm{H}} \mathbf{w}_{j m}^{c}\right|^{2}+N_{o} \\
\Psi & =\sum_{\substack{j \neq q \\
m \in \mathcal{U}_{j}^{c}}} P_{j m}^{c}\left|\mathbf{h}_{j q r}^{\mathrm{H}} \mathbf{w}_{j m}^{c}\right|^{2}+\sum_{m \in \mathcal{U}_{b}} P_{m}^{b}\left|\mathbf{g}_{b q r}^{\mathrm{H}} \mathbf{w}_{m}^{b}\right|^{2}+N_{o}
\end{aligned}
$$

able antennas at every given time slot. This issue is more pronounced when the number of UEs assigned to a cluster is greater than available antennas. This paper adopts proportional fair scheduling (PFS) and exploits the idea that given a fixed set of downlink beamforming vectors and power spectrum across the network, user scheduling at each cluster does not affect the interference seen by other UEs [2]. Therefore, each cluster can design a set of random, but fixed downlink beams independently and schedule UE $k^{*}$ for the current time if

$$
k^{*}=\arg \max _{k} \frac{R_{i k}^{c}}{\bar{R}_{i k}^{c}}
$$

where $R_{i, k}^{c}$ is computed as in (7) using the generated random beams and assuming equal power allocation across individual clusters. Furthermore, maximizing the proportional fair objective in (3) is equivalent to maximizing the weighted rate sum problem wherein weights are updated according to the time-averaged rates for each UE. Hence, the objective function in (3) can be modified as

$$
\operatorname{maximize} \sum_{i \in \mathcal{C}} \sum_{k \in \mathcal{U}_{i}^{c}} \gamma_{i k}^{c} R_{i k}^{c}+\sum_{k \in \mathcal{U}_{b}} \gamma_{k}^{b} R_{k}^{b}
$$

where $\gamma_{i k}^{c}$ and $\gamma_{k}^{b}$ are the weights associated with UE $k$ in cluster $i$ and macro UE $k$, respectively. Given a fixed set of scheduled UEs for each cluster and the macro BS, the optimal beamforming vectors can be designed.

\section{Zero-Forcing Beamforming}

In order to cancel intra-cluster interference, a linear ZFBF is employed within each cluster. The ZFBF precoding matrix of cluster $i \mathbf{W}_{i}^{c}$ and macro BS $\mathbf{W}_{b}$ are given as

$$
\begin{aligned}
& \mathbf{W}_{i}^{c}=\mathbf{H}_{i i}\left(\mathbf{H}_{i i}^{\mathrm{H}} \mathbf{H}_{i i}\right)^{-1} \\
& \mathbf{W}_{b}=\mathbf{H}_{b}\left(\mathbf{H}_{b}^{\mathrm{H}} \mathbf{H}_{b}\right)^{-1}
\end{aligned}
$$

where $\mathbf{H}_{i i}=\left[\mathbf{h}_{i i 1}, \ldots, \mathbf{h}_{i i K_{i}^{c}}\right] \in \mathbb{C}^{M_{i} \times K_{i}^{c}}$ and $\mathbf{H}_{b}=$ $\left[\mathbf{h}_{b 1}, \ldots, \mathbf{h}_{b K_{b}}\right] \in \mathbb{C}^{N \times K_{b}}$. Furthermore, each beam vector is normalized to have a unit norm, i.e., $\left\|\mathbf{w}_{i j}^{c}\right\|=\left\|\mathbf{w}_{k}^{b}\right\|=1$ for all $i, j$, and $k$.

\section{Coordinated Power Allocation}

Assuming a fixed set of scheduled UEs and downlink beam vectors, the final step of the algorithm is coordinated power allocation in order to maximize the weighted rate sum problem formulated in (3)-(6). However, this problem is non-convex and only local optimality can be guaranteed via KarushKuhn-Tucker (KKT) conditions. Consider the objective function (11), the dual problem can be stated as

$$
\begin{aligned}
& \mathcal{L}\left(P_{i k}^{c}, P_{k}^{b}, \lambda, \mu\right)=\sum_{i \in \mathcal{C}} \sum_{k \in \mathcal{U}_{i}^{c}} \gamma_{i k}^{c} R_{i k}^{c}+\sum_{k \in \mathcal{U}_{b}} \gamma_{k}^{b} R_{k}^{b}+ \\
& \sum_{i \in \mathcal{C}} \lambda_{i}^{c}\left(P_{i}^{c}-\sum_{k=1}^{K_{i}^{c}} P_{i k}^{c}\right)+\mu^{b}\left(P_{b}-\sum_{k=1}^{K_{b}} P_{k}^{b}\right)
\end{aligned}
$$

where $\lambda_{i}^{c}$ and $\mu^{b}$ are the Lagrangian multipliers corresponding to the sum power constraints in the original optimization problem. The amount of allocated power from cluster $i$ to its $k^{\text {th }}$ beam $P_{i k}^{c}$ and from the macro BS to its $k^{\text {th }}$ beam $P_{k}^{b}$ can be computed, respectively, via KKT conditions as

$$
\begin{aligned}
P_{i k}^{c} & =\left[\frac{\gamma_{i k}^{c}}{\lambda_{i}^{c}+t_{i k}^{c}}-\frac{I_{i k}^{c}+N_{o}}{\left|\mathbf{h}_{i i k}^{\mathrm{H}} \mathbf{w}_{i k}^{c}\right|^{2}}\right]^{+} \\
P_{k}^{b} & =\left[\frac{\gamma_{k}^{b}}{\mu^{b}+t_{k}^{b}}-\frac{I_{k}^{b}+N_{o}}{\left|\mathbf{h}_{b k}^{\mathrm{H}} \mathbf{w}_{k}^{b}\right|^{2}}\right]^{+}
\end{aligned}
$$

where $I_{i k}^{c}$ and $I_{k}^{b}$ denote the total interference power experienced by UE $k$ associated with the $i^{\text {th }}$ cluster and the macro BS, respectively. This information can be measured at each UE and fed back to its corresponding transmitter. $[x]^{+}=\max (0, x), t_{i k}^{c}$ and $t_{k}^{b}$ are defined in (9)-(10) and can be interpreted as "interference cost", which measure, respectively, the amount of interference that transmission of cluster $i$ and the macro BS over their $k^{\text {th }}$ beams impose on UEs of other cells [12]. With $t_{i k}^{c}, t_{k}^{b}, I_{i k}^{c}$, and $I_{k}^{b}$ fixed, each transmitter is able to distribute its power among its associated UEs only based on its local information. Then, the interference costs and total interference powers at each UE are updated based on the obtained transmit powers. This process is repeated until convergence. 
TABLE I

Network Design Parameters

\begin{tabular}{|c|c|}
\hline BS Max Tx power & $43 \mathrm{dBm}$ \\
\hline SCA Max Tx power & $24 \mathrm{dBm}$ \\
\hline Number of SCAs & $S=20$ \\
\hline Number of UEs & $K=100$ \\
\hline Number of BS antennas & $N=10$ \\
\hline Number of SCA antennas & $N_{i}=2$ \\
\hline Cell side length & $L=200$ \\
\hline Total bandwidth & $W=10 \mathrm{MHz}$ \\
\hline Background noise & $N_{o}=-174 \mathrm{dBm} / \mathrm{Hz}$ \\
\hline BS-to-UE shadowing & $\sigma_{i j}=6 \mathrm{~dB}$ \\
\hline SCA-to-UE shadowing & $\sigma_{i j}=4 \mathrm{~dB}$ \\
\hline
\end{tabular}

Finally, the achievable rates of UEs can be computed during a given time slot $t$ using (7)-(8). Moreover, the scheduling weights of UEs are updated as $\gamma_{i k}^{c}=1 / \bar{R}_{i k}^{c}(t)$ and $\gamma_{k}^{b}=$ $1 / \bar{R}_{k}^{b}(t)$ wherein

$$
\begin{aligned}
\bar{R}_{i k}^{c}(t) & =\beta \bar{R}_{i k}^{c}(t-1)+(1-\beta) R_{i k}^{c}(t) \\
\bar{R}_{k}^{b}(t) & =\beta \bar{R}_{k}^{b}(t-1)+(1-\beta) R_{k}^{b}(t)
\end{aligned}
$$

and $0<\beta<1$ is a forgetting factor.

The proposed approach is reminiscent of [2] which solves the scheduling, beamforming, and power spectrum management in an iterative fashion. In particular, we observe that once a beam is assigned to a UE during the first iteration, it will likely not be assigned to a different UE during later iterations. Hence, our non-iterative approach reaches about the same local solution as that of the iterative scheme.

\section{Simulation Results}

This section evaluates the performance of the proposed algorithm on a single-cell wireless network augmented with $S=20$ SCAs. Both tiers transmit simultaneously over the available bandwidth $W=10 \mathrm{MHz}$. The macro BS is equipped with $N=10$ antennas and SCAs employ two antennas each. Furthermore, $K=100$ UEs are uniformly scattered within a square cell region of area $200 \times 200 \mathrm{~m}^{2}$. The distance dependent channel model between any pair of nodes is modeled as $28.1+37.6 \log _{10} d_{i j}+\chi_{i j}$ in $\mathrm{dB}$ wherein $d_{i j}$ is the distance between node $i$ and $j$ and $\chi_{i j}$ denotes lognormal shadow fading with standard deviation of $\sigma_{i j} \mathrm{~dB}$. The simulation results are averaged over node locations and channel realizations. The system parameters are outlined in Table I.

We investigate the performance of a two-tier network where SCAs act independently (per-cell processing), form clusters using $k$-means or AP-clustering schemes, and form one very large virtual MIMO system (global coordination) with equal power allocation (EPA) and the proposed coordinated power allocation scheme (CPA). As Fig. 2 suggests, a network employing the proposed CPA scheme always outperforms EPA transmission strategy. Moreover, since the number of interfering sources decreases with clustering, CPA with clustering provides additional performance gain compared to all three schemes with EPA and also per-cell processing with CPA. In particular, it provides $75 \%$ and $55 \%$ rate improvements for

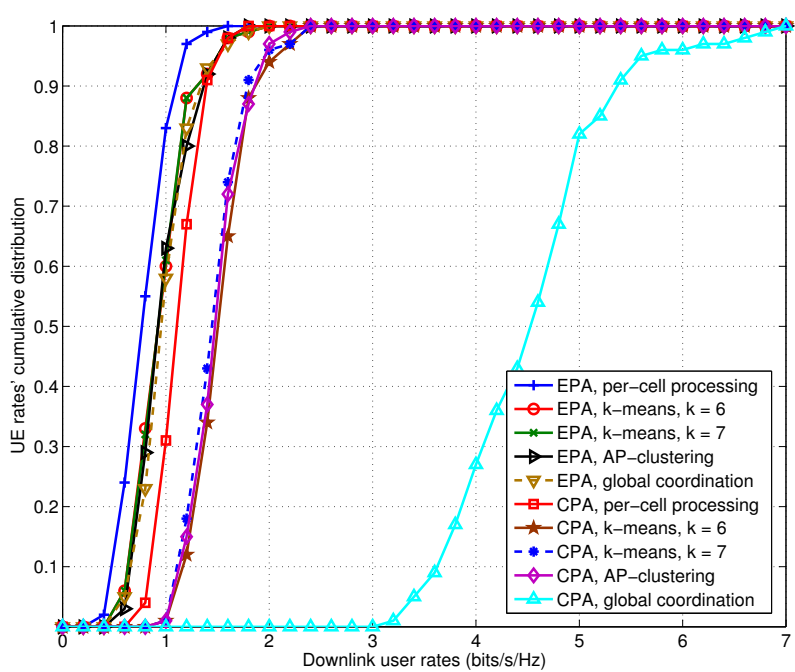

Fig. 2. Cumulative distribution of user rates for different schemes with Equal Power Allocation (EPA) and Coordinated Power Allocation (CPA).

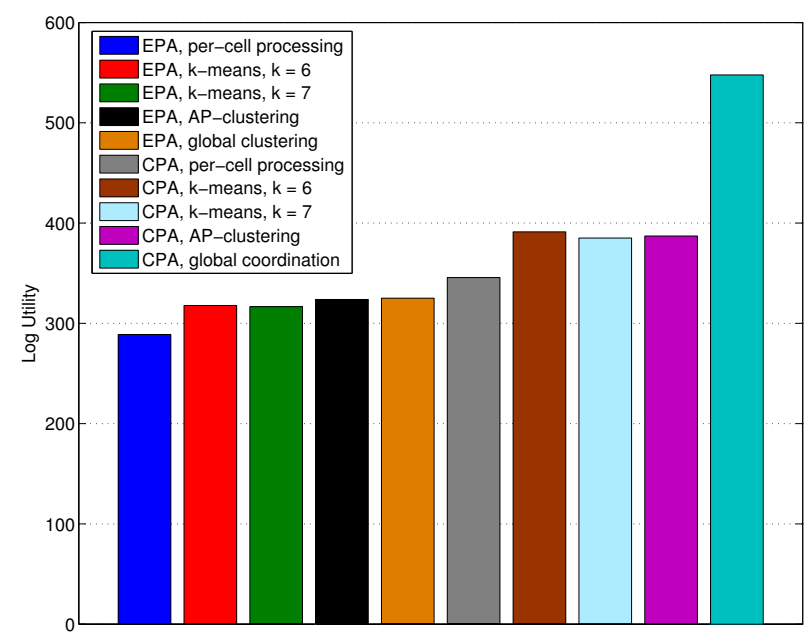

Fig. 3. Log utility gain of different clustering schemes with EPA and CPA. Log utilities are computed with rates in Mbps.

$20^{\text {th }}$ percentile UEs compared to global coordination with EPA and per-cell processing with CPA, respectively.

Fig. 3 and 4 illustrate the log utility gains and required backhaul capacity ${ }^{3}$ of different schemes. As can be seen from the figures, global coordination with CPA provides $170 \%$ rate enhancements for $20^{\text {th }}$ percentile UEs and $30 \%$ improvement in $\log$ utility across the network. However, this gain comes at a price of requiring almost $1000 \%$ more backhaul capacity. Moreover, the performance of the global coordination is unrealistic, as in any practical deployment, there will always

\footnotetext{
${ }^{3}$ The required backhaul capacity for each user is calculated as its achievable rate times the number of its serving access points.
} 


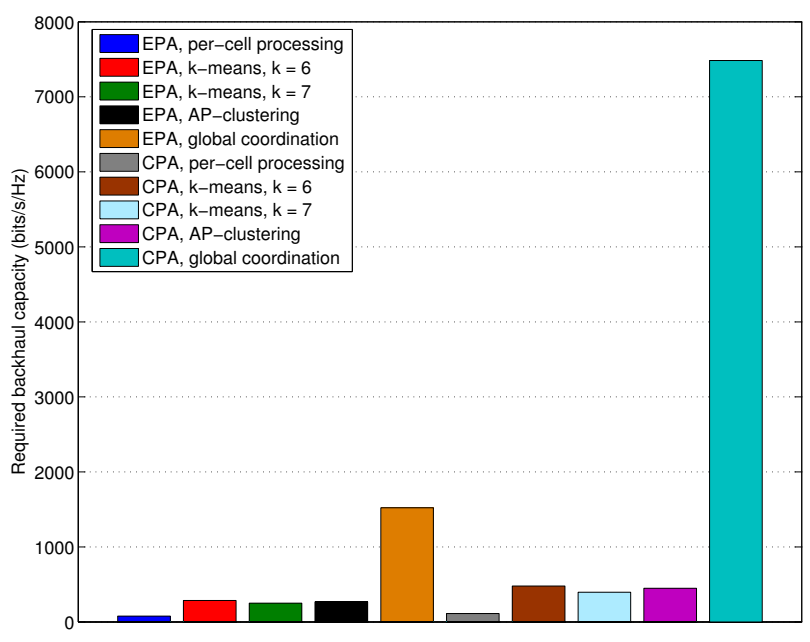

Fig. 4. Required backhaul capacity of different clustering schemes with EPA and CPA.

TABLE II

Performance Comparison of Three Clustering Schemes

\begin{tabular}{|c|c|c|c|}
\hline Algorithm & Log Utility & Backhaul Capacity & Variance \\
\hline \hline AP & 387.07 & 424 & 1.32 \\
\hline$k$-means, $k=6$ & 391.31 & 452.46 & 1.93 \\
\hline$k$-means, $k=7$ & 385.03 & 370.60 & 1.89 \\
\hline SP, $k=6$ & 395.29 & 656.12 & 4.25 \\
\hline SP, $k=7$ & 387.26 & 544.82 & 4.10 \\
\hline
\end{tabular}

be out-of-cell interference. Therefore, SC clustering via either $k$-means or AP-clustering scheme concatenated with CPA can be considered as a reasonable alternative approach for future wireless networks.

Table II evaluates the performance of AP-clustering and $k$ means with respect to log utility, required backhaul capacity (bits/s/Hz), and the variance of obtained cluster sizes ${ }^{4}$ against SP-clustering. For AP-clustering, the self-similarity values are set as the minimum value of mutual similarities. This corresponds to generating more populated clusters. In this case, the average number of clusters is 6.2. Although SPclustering achieves the highest log-utility, it requires high data-rate backhaul links. The reason is, as the cluster size variances confirm, the obtained clusters via SP-clustering are non-homogeneous, i.e., a few number of clusters include most of the active SCAs, while the remaining SCAs form single-member clusters. However, AP-clustering and $k$-means performances are almost identical.

\section{CONCLUSION}

This paper investigates a cluster-based coordinated intercell interference management scheme for a downlink of heterogeneous networks wherein transmitters are equipped

\footnotetext{
${ }^{4}$ For fixed node locations, variance is computed as the difference between the largest cluster size and the average cluster size.
}

with multiple antennas. The proposed AP-clustering and $k$ means cluster formation processes are merely functions of the network topology; hence, they are stable over long time scales. The key point here is that clustering facilitates partial interference cancellation in dense deployments in an efficient manner. In particular, UEs' data symbols are merely shared amongst a small fraction of active SCAs. Moreover, channel training is only required within each cluster. Each cluster forms a virtual MIMO network system which serves its associated UEs via zero-forcing spatial multiplexing. Moreover, a simple interference pricing power allocation is devised to reduce the intercell interference without exchanging UEs' data symbols. The proposed clustering scheme concatenated with coordinated power allocation provides significant networkutility improvements compared to the conventional per-cell processing. Furthermore, it requires significantly less backhaul communication and channel training overhead as compared to the global coordination scheme.

\section{REFERENCES}

[1] J. G. Andrews, H. Claussen, M. Dohler, S. Rangan, and M. Reed, "Femtocells: Past, present, and future," IEEE J. Sel. Areas Commun., vol. 30, no. 3, pp. 497-508, Apr. 2012.

[2] W. Yu, T. Kwon, and C. Shin, "Multicell coordination via joint scheduling, beamforming, and spectrum adaptation," Proc. IEEE INFOCOMM, pp. 2570-2578, Apr. 2011.

[3] C. T. K. Ng and H. Huang, "Linear precoding in cooperative MIMO cellular networks with limited coordination clusters," IEEE J. Select. Areas Commun., vol. 28, no. 9, pp. 1446-1454, Dec. 2010.

[4] L. Venturino, N. Prasad, and X. Wang, "Coordinated linear beamforming in downlink multi-cell wireless networks," IEEE Trans. Wireless Commun., vol. 9, no. 4, pp. 1451-1461, Apr. 2010.

[5] B. Golkar and E. Sousa, "Adaptive partitioned interference management in cellular networks," Proc. IEEE GLOBECOM, pp. 4477-4482, Dec. 2008.

[6] K. Hosseini, H. Dahrouj, and R. S. Adve, "Distributed clustering and interference management in two-tier networks," Proc. IEEE GLOBECOM, pp. 4483-4488, Dec. 2012.

[7] M. Hong, R. Sun, H. Balighi, and Z. Q. Luo, "Joint base station clustering and beamformer design for partial coordinated transmission in heterogeneous networks," 2012. [Online]. Available: http://arxiv.org/abs/1203.6390

[8] R. Duda, P. Hart, and D. Stork, Pattern Classification. John Wiley \& Sons, 2000.

[9] U. Luxburg, "A tutorial on spectral clustering," Statistics and Computing, vol. 17, Dec. 2007.

[10] B. J. Frey and D. Dueck, "Clustering by passing messages between data points," Science, vol. 315, no. 5814, pp. 972-976, 2007.

[11] W. Saad, Z. Han, M. Debbah, A. Hjorungnes, and T. Basar, "Coalitional game theory for communication networks," IEEE Signal Proc. Mag., pp. 77-97, Sep 2009.

[12] W. Yu, "Multiuser water-filling in the presence of crosstalk," in Proc. Information Theory and Applications Workshop (ITA'07), 2007, pp. 414 420.

[13] F. R. Kschischang, B. J. Frey, and H. A. Loeliger, "Factor graphs and the sum-product algorithm," IEEE Trans. Inform. Theory, vol. 47, no. 2, pp. 498-519, Feb. 2001. 